\title{
NOTE
}

\section{Destriping LANDSAT MSS Images by Histogram Modification}

\author{
Berthold K. P. Horn and Robert J. Woodham \\ Artificial Intelligence Laboratory, Massachuselts Institute of Technology, \\ Cambridge, Massachusetts, 02189
}

Received May 3, 1978; accepted August 21, 1978

\begin{abstract}
Before images obtained by multisensor cameras can be used in image analysis, corrections must be introduced for the differences in the gain or transfer functions of the sensors. Methods are here presented for obtaining the required information directly from the statistics of the sensor outputs. The assumption is made that the probability distribution of scene radiances seen by each sensor is the same. Successful destriping of LANDSAT Multispectral Scanner (MSS) images is demonstrated. The technique applies to images obtained with any multisensor line-scan camera, however.
\end{abstract}

\section{DESTRIPING OF IMAGES OBTAINED USING MULTIPLE SENSORS}

An image-sensing device using a single photoelectric sensor which is mechanically scanned across the scene produces outstanding digitized images, since sensitivity, resolution, and gain or transfer function are the same for all points in the image. Unfortunately, such a device is limited in speed by the mechanical movement. More importantly, it is limited in speed by the fact that an accurate measurement of scene radiance requires the collection of an adequate number of photons. This explains the preponderance of linear arrays of sensors and area sensors such as vidicons which are otherwise deficient because of geometric distortions, nonuniform response, nonuniform resolution, and so on.

A compromise can be struck, where a small set of sensors is mechanically scanned to collect the image. In the system used aboard LANDSAT, for example, each spectral band is scanned using six sensors at the same time. Thus, six lines of the image are produced during a single sweep of the mirror. On the next sweep the satellite has advanced in its orbit by an amount which allows the same set of sensors to pick up the next six lines of the image [1].

Unfortunately, the sensors do not have identical gain functions. As a result, images produced in this fashion show undesirable, regular "striping." This effect could be removed if the gain functions were accurately known, since one could then compute scene radiance from the sensor output using the inverses of these gain functions. The sensors used in older equipment in particular have 
time-varying behavior. Photomultipliers, for example, show a drift in both gain and offset (dark current) due to small changes in the material of the dynodes used in the electron multiplier stages and temperature variations.

If a reference object containing all scene radiances of interest were in the scene, one could recalibrate the sensors continuously. This is difficult to arrange. An alternative is the scanning of a gray wedge placed over a light source at the end of every scan line. This, in fact, is what is done aboard LANDSAT. The results are used to estimate the gains and offsets of the sensors. The digital data produced from the raw satellite signals is corrected using this information [1].

Unfortunately, one finds that the striping effect is not removed in this fashion; the reasons for this are not entirely clear. One cause appears to be the use of the calibration data as a means of adjusting gain and offset so that each sensor is related to its preflight condition. Slight changes in the light source, the gray wedge, and the geometry of imaging introduce drifts which are not compensated for. Another reason is related to the fact that photomultipliers are somewhat nonlinear and have a response which depends on their exposure history. Modern devices using solid state photodiodes do not suffer from these problems.

The methods explored here for destriping images are based on the assumption that each sensor is exposed to scene radiances with approximately the same probability distribution. The sensor values can then be modified so that each one is related in the same way to the actual scene radiance. The information required to perform this modification is extracted from statistics of the observed sensor outputs.

\section{A SIMPLE METHOD FOR LINEAR TRANSDUCERS}

If the image sensors are linear and time invariant, a simple method can be used to reduce striping. The sensor output, $x^{\prime}$, can be written as a function of the scene radiance, $x$, as follows:

$$
x^{\prime}=f(x)=a+b \cdot x .
$$

Each sensor has its own, fixed values of offset, $a$, and gain, $b$. If these are known, the scene radiance can be calculated using the inverse of the transfer function,

$$
x=g\left(x^{\prime}\right)=\left(x^{\prime}-a\right) / b .
$$

If this is done for each sensor in turn, striping effects will be removed.

The required constants for each sensor can be determined if a calibration object containing two or more known scene radiance values is available in the scanned scene. If such a calibration object is not available one can estimate the (relative) values of gain and offset using simple statistics of observed sensor values. Each sensor sees a subimage consisting of every $n$th line (when $n$ sensors are used). The complete image is formed by interlacing these subimages. It seems reasonable to suppose that, for a large enough image, each subimage has approximately the same probability distribution of scene radiance values. One would not expect a particular subimage to contain many more values in a particular range of scene radiances than another subimage. 
If this assumption is correct, then the gain and offset constants can be estimated from the mean and standard deviation of the measured sensor output values. If the mean of the scene radiance is $\mu$ and the standard deviation is $\sigma$, then the mean of the sensor output will be $\mu^{\prime}=a+b \mu$, and the standard deviation of the sensor output $\sigma^{\prime}=b \sigma$. Then,

$$
b=\sigma^{\prime} / \sigma
$$

and

$$
a=\left(\mu^{\prime} \sigma-\mu \sigma^{\prime}\right) / \sigma
$$

Clearly, it is not reasonable to assume that one can find the absolute values of the mean and standard deviation of the actual scene radiance. Fortunately, for destriping purposes only relative values are important. That is, one can use the mean and standard deviation of the sensor outputs for the whole image in place of the mean and standard deviation of the scene radiance. Naturally now the results will not be scene radiance values. The striping, however, will be removed since each subimage now has the same mean and standard deviation, and, if the assumption introduced earlier applies, the same linear relationship to scene radiance.

Note that one can relax the assumption about the relationship of the subimages. Here it is not necessary that they have the same probability distribution of scene radiance, only that their means and standard deviations be the same. This simple method has been applied by some users of LANDSAT data [2, 3$]$.

\section{SHORTCOMINGS OF THE SIMPLE METHOD}

We have found this method to be only partially successful in destriping LANDSAT images. One reason for this may be that out of a range of 128 possible sensor outputs a range of only around 30 values correspond to normal scene radiance values. Low values are not found in short wavelength bands because of light scatter in the air. Conversely, large values correspond to cloud, snow, and ice, and scene radiance values of such areas often exceed the highest available sensor output values and so result in clipping. Clipping of sensor values corresponding to low scene radiances also occurs at times in the long wavelength bands due to negative sensor offsets. Both of these nonlinear effects will introduce skew into the calculation of means and standard deviations.

One may alleviate this problem by removing sensor values outside a certain range from consideration. While slightly better results are obtained in this fashion, it is clear that the arbitrarily selected thresholds needed introduce biases of their own. This later effect can be dealt with by eliminating the same fraction of sensor values from the low end of the output of each sensor. Similarly, a fixed fraction of sensor values is removed from the high end.

Even with this refinement, results are not entirely satisfactory. Superficially, it appears that different gains and offsets are appropriate for different scene radiance ranges. That is, the sensor transfer curves are somewhat nonlinear. We thus devised a method which deals with this problem directly. 


\section{ANALYSIS OF CONTINUOUS TRANSDUCER}

Consider a transducer which produces an output $x^{\prime}$ when presented with an input $x$, where $x^{\prime}=f(x)$. Suppose that a random variable $X$ with probability density function $p(x)$ is applied to the input of the transducer. The resulting output can be thought of as a new random variable $X^{\prime}$, say, with probability density function $p^{\prime}\left(x^{\prime}\right)$. To find the relationship between the two probability density functions it is helpful to introduce the cumulative probability density functions $P(x)$ and $P^{\prime}\left(x^{\prime}\right)$, where

and

$$
P(x)=\int_{-\infty}^{x} p(t) d t
$$

$$
P^{\prime}\left(x^{\prime}\right)=\int_{-\infty}^{x^{\prime}} p^{\prime}(t) d t .
$$

If $f(x)$ is monotonically nondecreasing and $x^{\prime}=f(x)$, it is easy to show that $P^{\prime}\left(x^{\prime}\right)=P(x)$. Consequently one can determine $f(x)$ if the cumulative probability density functions $P(x)$ and $P^{\prime}\left(x^{\prime}\right)$ are known and if the latter has an inverse. Then

$$
f(x)=\left(P^{\prime}\right)^{-1} P(x) .
$$

The required inverse will exist if $P^{\prime}\left(x^{\prime}\right)$ is monotonically increasing.

To calculate scene radiance from sensor values we actually need the inverse $g\left(x^{\prime}\right)$ of the gain or transfer function $f(x)$. This function can be found just as easily, since $x=g\left(x^{\prime}\right)$ implies $P(x)=P^{\prime}\left(x^{\prime}\right)$. Evidently one can determine $g\left(x^{\prime}\right)$ if the cumulative probability density functions $P^{\prime}\left(x^{\prime}\right)$ and $P(x)$ are known and if the latter has an inverse. Then,

$$
g\left(x^{\prime}\right)=(P)^{-1} P^{\prime}\left(x^{\prime}\right) .
$$

The method shown here for finding the gain function of the transducer is based on the same analysis as that used to design a generator of pseudorandom numbers with desired probability distribution function $p^{\prime}\left(x^{\prime}\right)$, when a generator is available which produces pseudorandom numbers with known probability distribution function $p(x)[4]$.

A graphical illustration of the relationships discussed may be found in Fig. 1. The dotted line suggests how one may determine the transducer output value $f(x)$ given the scene radiance value, $x$. Conversely, the same dotted line may be followed in the reverse direction to find the value $g\left(x^{\prime}\right)$ of scene radiance from a given value of the transducer output, $x^{\prime}$.

\section{TRANSDUCER WITH DISCRETE OUTPUT VALUES}

Essentially the same method may be used if the transducer produces discrete outputs. Consider, for example, a case where the input range can be broken up into a number of intervals such that

$$
f(x)=i \quad \text { if } x \in\left[x_{i}, x_{i+1}\right) .
$$



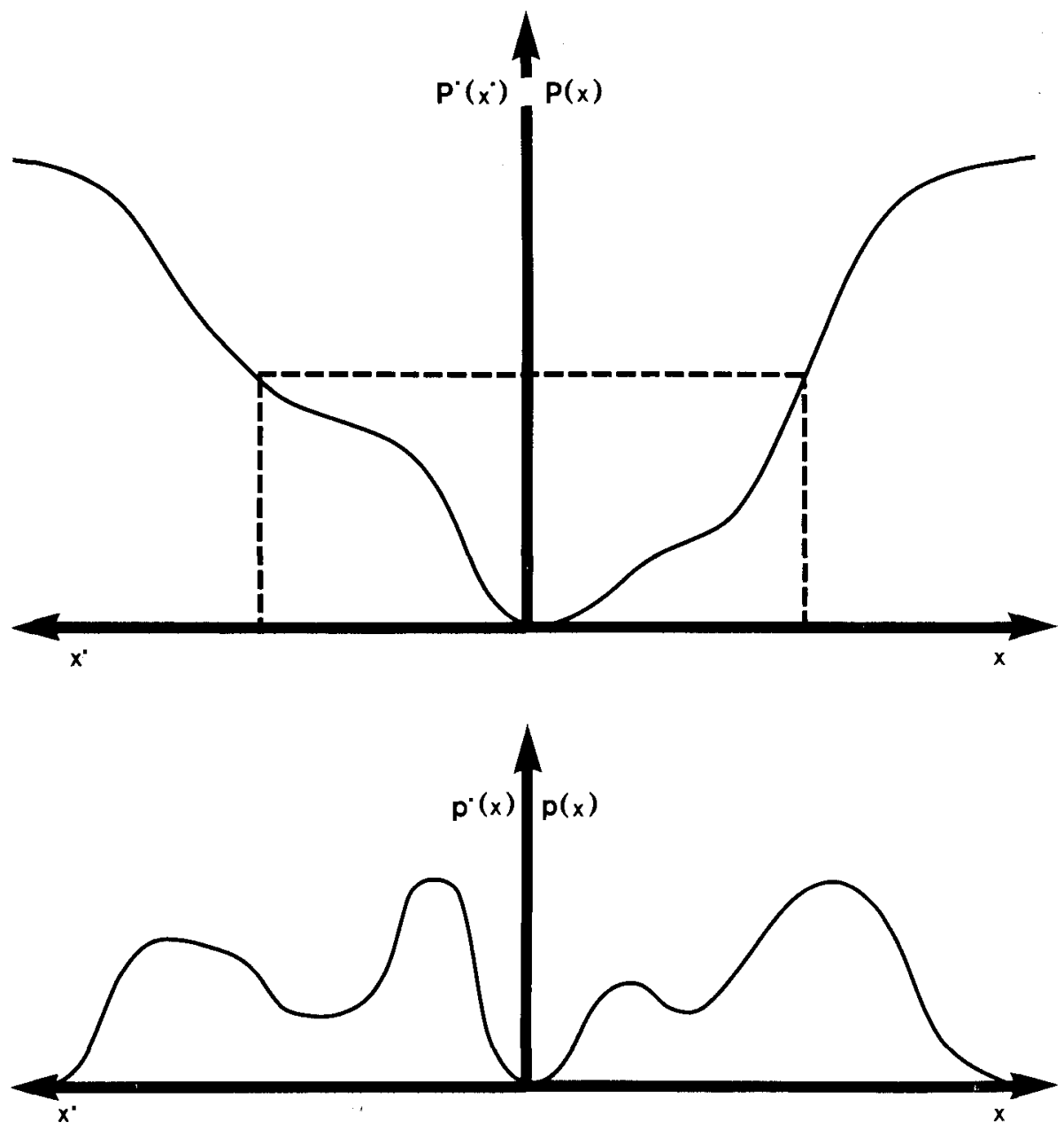

Fig. 1. The transfer function, $x^{\prime}=f(x)$, of a transducer can be found if the cumulative probability distribution functions of its input, $x$, and its output, $x^{\prime}$, are known.

The probability density function of the output of the transducer is then discrete, and

Clearly, $p^{\prime}{ }_{i} \geq 0$ and

$$
p_{i}^{\prime}=\lim _{\epsilon \rightarrow 0} \int_{x_{i}}^{x_{i+1}-\epsilon} p(x) d x .
$$

$$
\sum_{i=-\infty}^{\infty} p_{i}^{\prime}=1
$$

The cumulative probability density function can be defined as follows:

$$
P_{i}^{\prime}=\sum_{a=-\infty}^{i} p_{a}^{\prime}
$$


If $f(x)$ is monotonically nondecreasing and $i=f(x)$, then a similar argument to the one used in the continuous case leads to $P^{\prime}{ }_{i}=P(x)$. If $P^{\prime}$ can be inverted, the transfer function can be found using

$$
f(x)=\left(P^{\prime}\right)^{-1} P(x) .
$$

The only difference is that here $f(x)$ is a function from a continuous range to a discrete domain. Naturally, when one finds the inverse of the transfer function, $g\left(x^{\prime}\right)$, using these methods, one has to accept the fact that the actual value of $x$ cannot be recovered, only a range $\left[x_{i}, x_{i+1}\right)$.

$$
g\left(x^{\prime}\right)=(P)^{-1} P^{\prime}\left(x^{\prime}\right)
$$

\section{APPLICATION TO SATELLITE IMAGES}

A particular sensor, in a system using $n$ sensors, sees a subimage containing every $n$th line. To apply the methods developed here one has to assume that each sensor is exposed to scene radiances with similar probability distributions. If the image is large enough, it is very unlikely that one sensor will see substantially fewer or more scene radiances in a particular range. A sensor's properties can then be estimated from the statistics of its outputs.

Since the probability distribution function of the actual scene radiance is not available, only relative adjustments can be made. That is, the probability distribution function of sensor outputs for the whole image is used as a reference. Consequently, application of the inverse functions so determined to each subimage will not produce scene radiances. It will, however, result in an image in which each image gray level is related to the scene radiance in the same way. Thus, striping will have been removed.

A graphical illustration of the method is shown in Fig. 2. Note that here both $P(x)$ and $P^{\prime}\left(x^{\prime}\right)$ are discrete. This results in a small problem when the transfer function $f(x)$ is to be found using data from a real image. For perfect data, there always is a value $x^{\prime}$ for every value $x$, such that $P^{\prime}\left(x^{\prime}\right)=P(x)$. As indicated by the dotted line in the figure, this may not be the case when the data is obtained from a finite number of samples obtained by different sensors. The best one can do then is to find a value $x^{\prime}$ such that

$$
P^{\prime}\left(x^{\prime}\right) \leq P(x)<P^{\prime}\left(x^{\prime}+1\right) .
$$

Similarly, in determining the inverse transfer function $g\left(x^{\prime}\right)$, one can do no better than finding a value $x$ such that

$$
P(x) \leq P^{\prime}\left(x^{\prime}\right)<P(x+1) .
$$

What value should be used in the lookup table for destriping? One might argue that some values should be translated to $x$, others to $(x+1)$. If this is done in the appropriate fashion, the histogram of gray levels in the destriped image will equal the histogram of gray levels in the raw image. It is difficult, however, to decide which points should receive one value and which the other. If the selection is based on a random number generator, additional noise will be introduced. In any case a change smaller than one gray level is usually negligible considering 


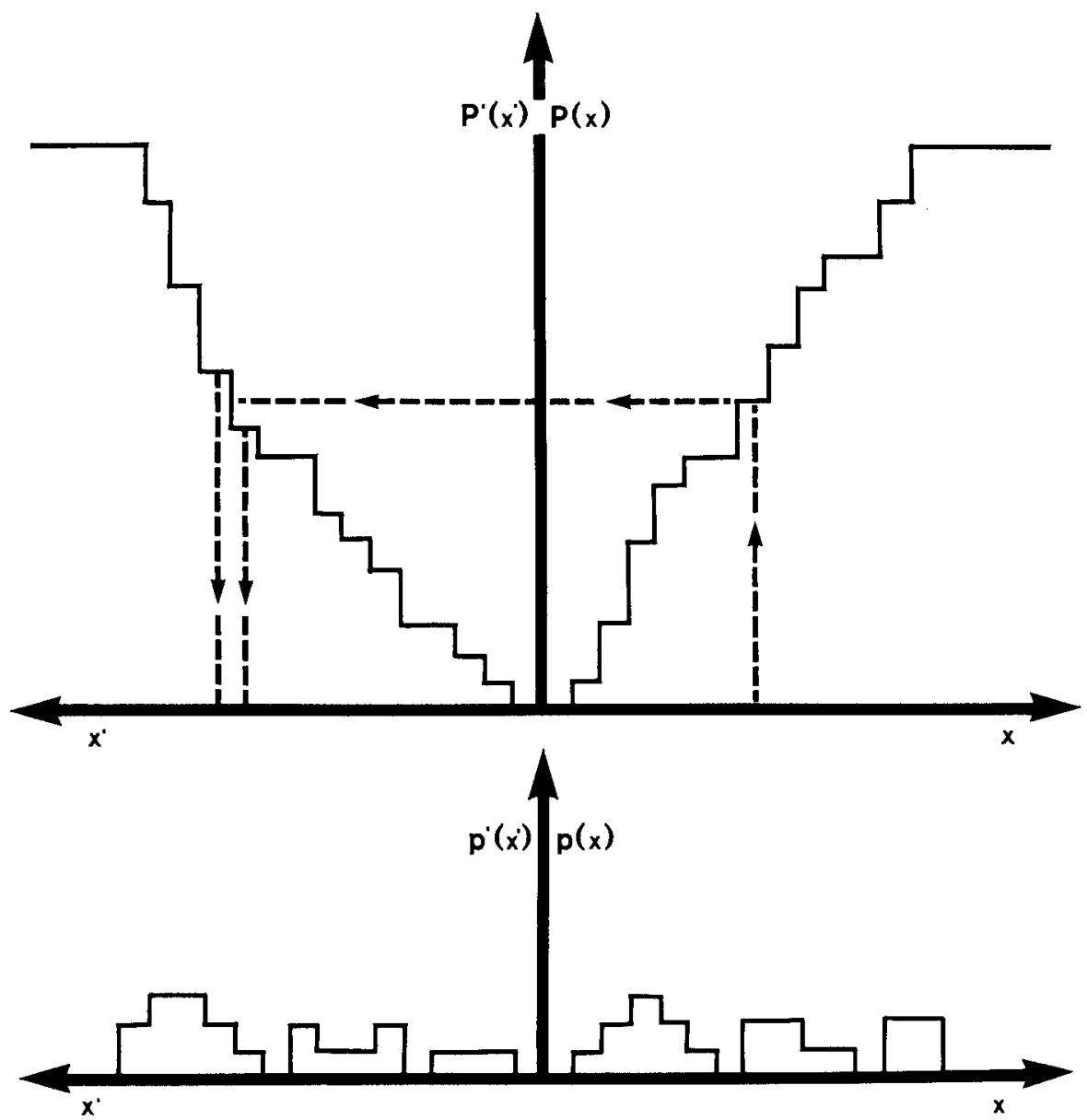

Fig. 2. When both cumulative probability distribution functions are discrete, there may not be a value of $x^{\prime}$ such that $P\left(x^{\prime}\right)=P(x)$. Some rule must be adopted to deal with this.

the limitations of radiometric accuracy in the imaging system. The algorithm we employed arbitrarily uses the smaller value of the two possible ones. Note that the histogram of the destriped image will then not be exactly equal to the histogram of the raw image.

\section{DETAILS OF THE ALGORITHMS}

The first step is the determination of a cumulative histogram of sensor values for the whole image as a reference. Let there be $H(x)$ occurrences of sensor outputs less than or equal to $x$ out of a total of $N$ values. Now for the subimage produced by sensor $i$, one calculates a similar cumulative histogram. Let $H_{i}\left(x^{\prime}\right)$ be the number of sensor outputs less than or equal to $x^{\prime}$, produced by sensor $i$, out of a total of $N_{i}$ values. Here

$$
N=\sum_{i=1}^{n} N_{i}
$$

where $n$ is the number of sensors. 
A lookup table $g\left(x^{\prime}\right)$ is now construeted by applying the inverse of the function $H(x)$ to $H_{i}\left(x^{\prime}\right)$. This lookup table is then used to modify all the sensor values produced by sensor $i$. The inverse can be calculated relatively easily since $H(x)$ is a monotonically nondecreasing function. The lookup table value $g\left(x^{\prime}\right)$ is the smallest number $x$ such that

$$
N_{i} H(x) \leq N H_{i}\left(x^{\prime}\right)<N_{i} H(x+1) .
$$

This process is repeated for each sensor in turn, until all image values have been modified by the lookup table appropriate to the sensor with which they were measured.

\section{RESULTS}

The image used for the illustrations here is a part of LANDSAT-1 image E-1078-09555 taken October 9, 1972. The part used contains 364 lines each of 430 pixels. The worst striping in this case occurred in band $6(0.7$ to $0.8 \mu)$ and band $7(0.8$ to $1.1 \mu)$, so the discussion will concentrate on these two. The data transmitted from the spaceeraft is pseudologarithmically compressed and con-

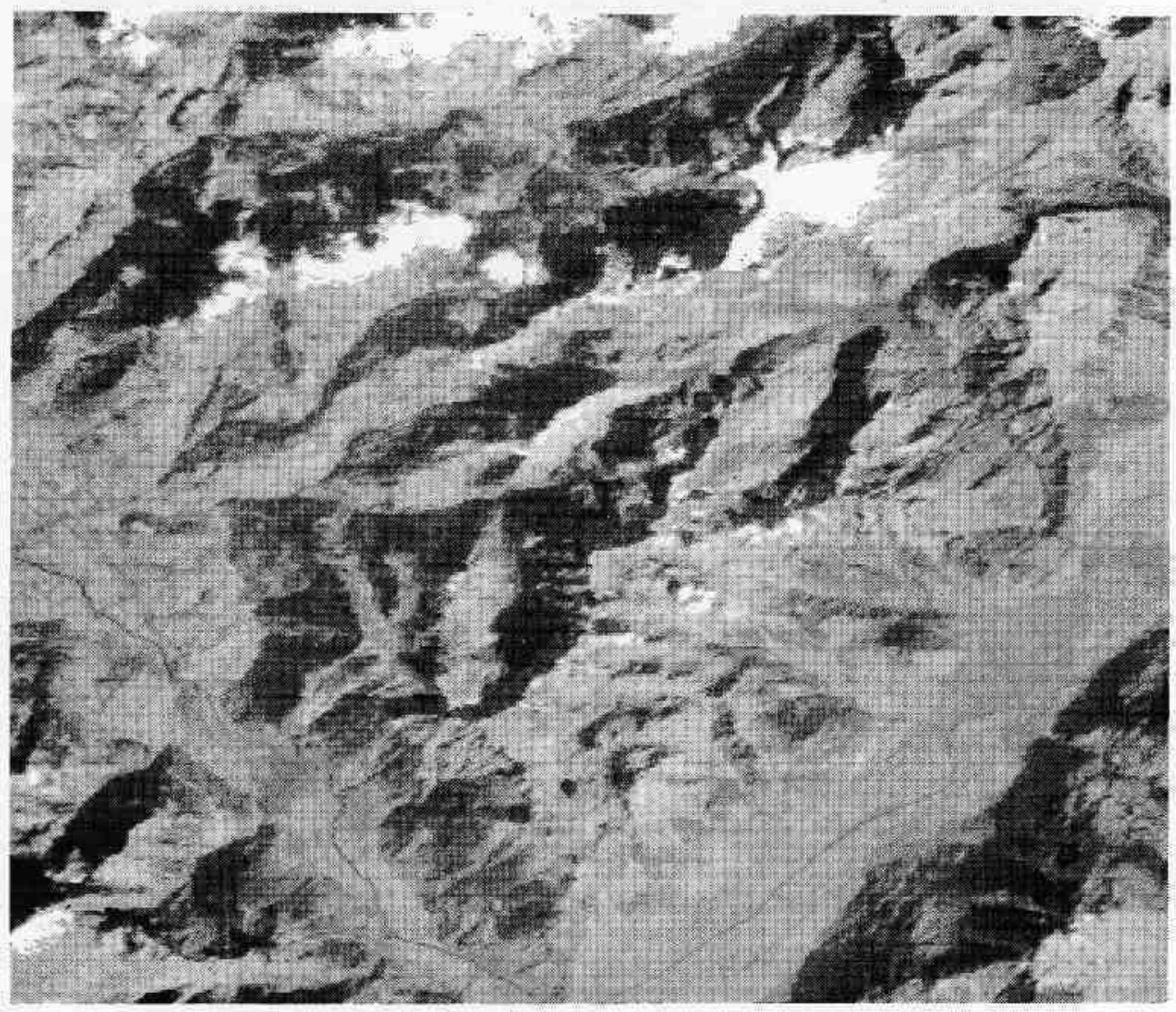

Fig. 3. Original, unreetified image of band $6(0.7$ to $0.8 \mu)$ output of LANDSAT. Note the heavy, regular striping. 


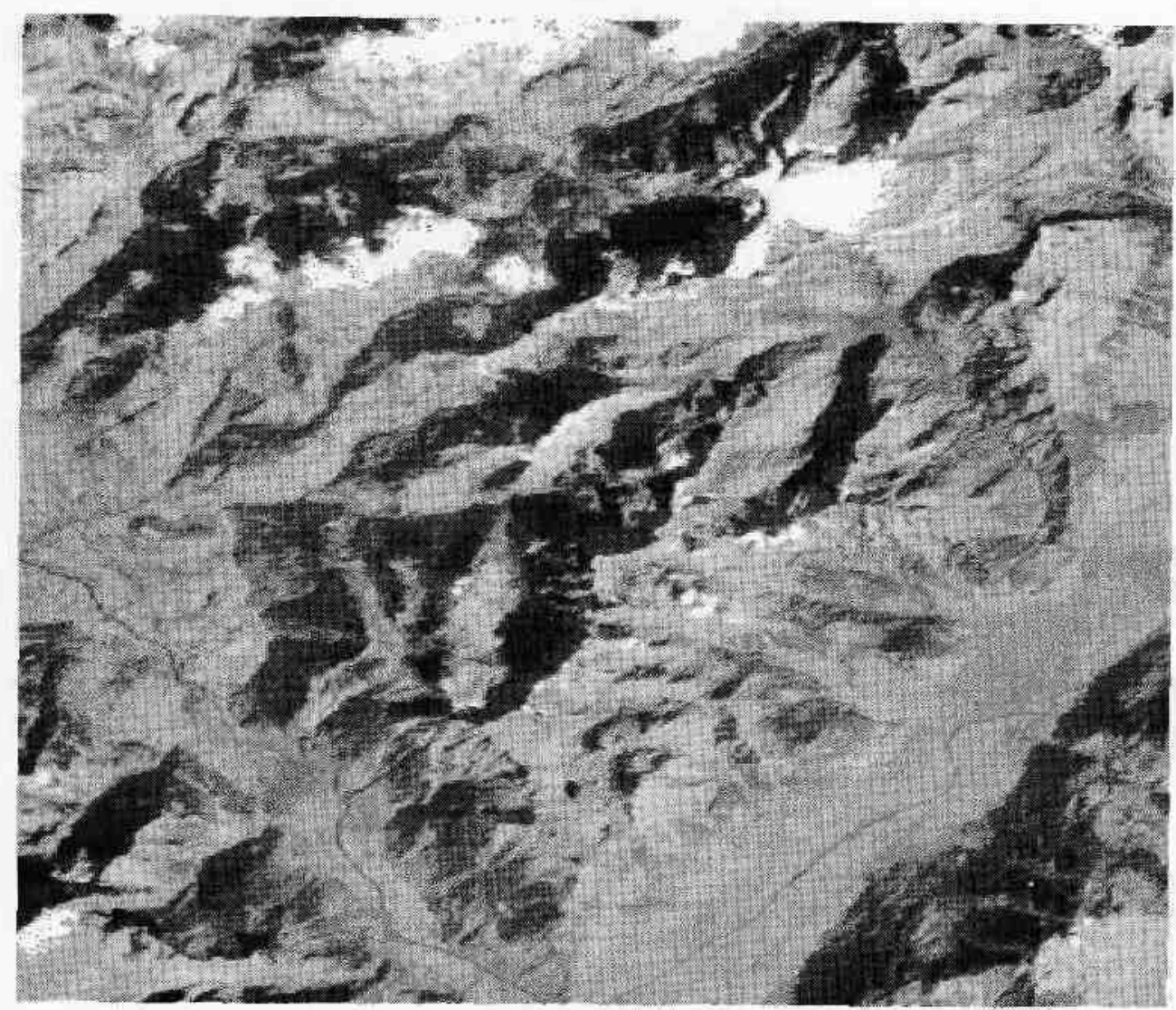

F1G. 4. Destriped image of band 6 output.

verted to six-bit numbers. On the ground the compression is removed using a lookup table. The result is a seven-bit number. Naturally, about half of the possible seven-bit numbers never oecur in a given subimage. Furthermore, ecene radiance corresponding to normal surfaces (other than ice, snow, or cloud) occupy only the lower 25 to $35 \%$ of the available range. As a result, the total range of scene radiances of interest corresponds to a very small set of distinct sensor output values. This contributes a little to difficulties in completely removing striping effects.

Comparison of Fig. 3, the original band 6 image, with Fig. 4, the processed version, shows that the heavy, regular striping is removed by the processing described here. It is instructive to inspect the lookup tables used for each sensor. These are shown as six subfigures in Fig. 5. The short horizontal sections in the transfer function correspond to sensor values which never occur as a result of the decompression table lookup. The inverse transfer functions shown in Fig. 5 appear to evidence some nonlinearities. This explains why the simple destriping technique described earlier does not do as well.

Comparison of Fig. 6, the original band 7 image, with Fig. 7 , the processed version, shows that the regular striping is removed here as well. The very light 
a.

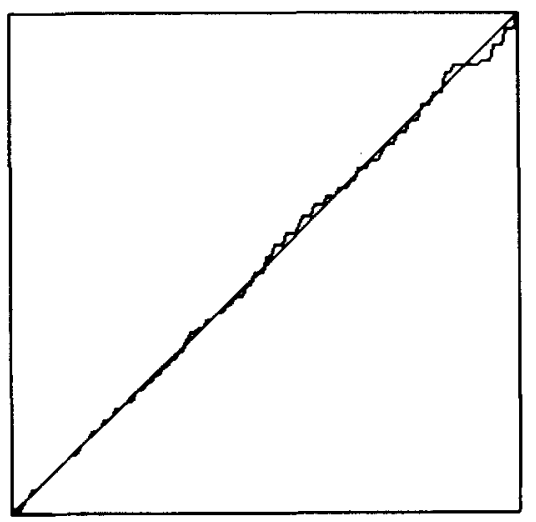

c.

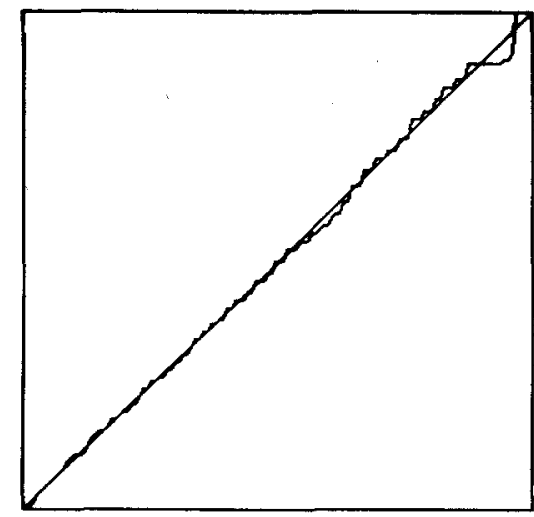

e.

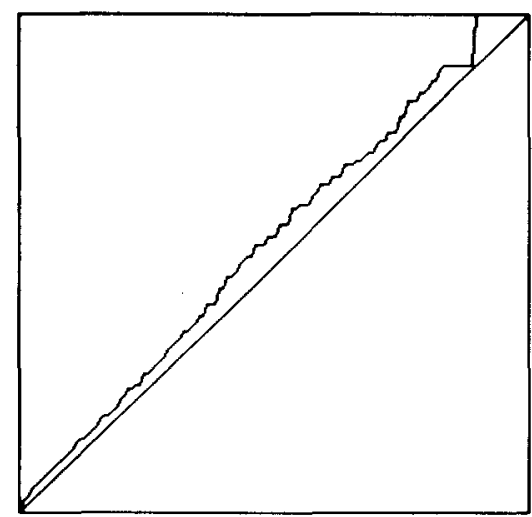

b.

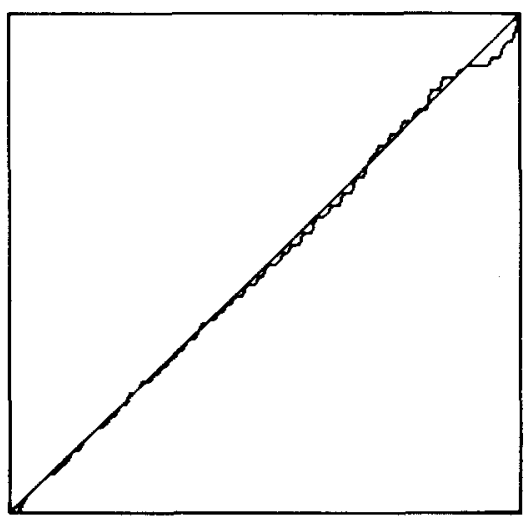

d.

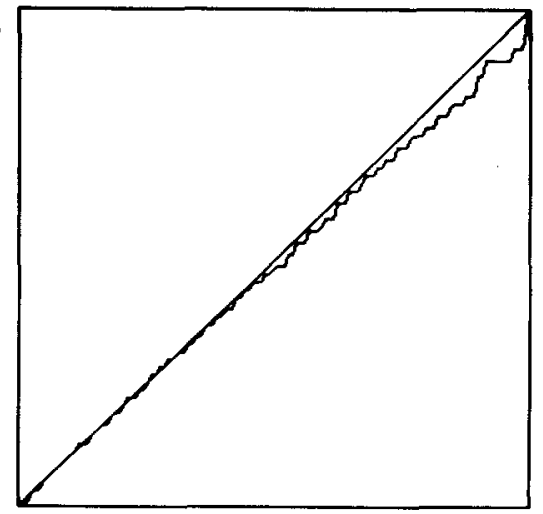

f.

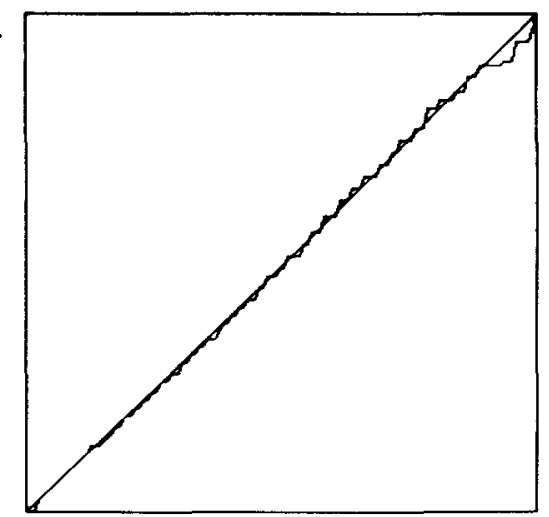

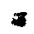

$\$ 9$

FIG. 5. The destriping lookup tables for band 6-inverse transfer functions for the six image sensors. The nonlinearities of the transducers are apparent.

striping remaining in shadow areas amounts to fluctuations of only one or two pixel values, but may be discernable because of the human observer's sensitivity to small differences of reflectance in dark areas. Inspection of the histograms of raw 


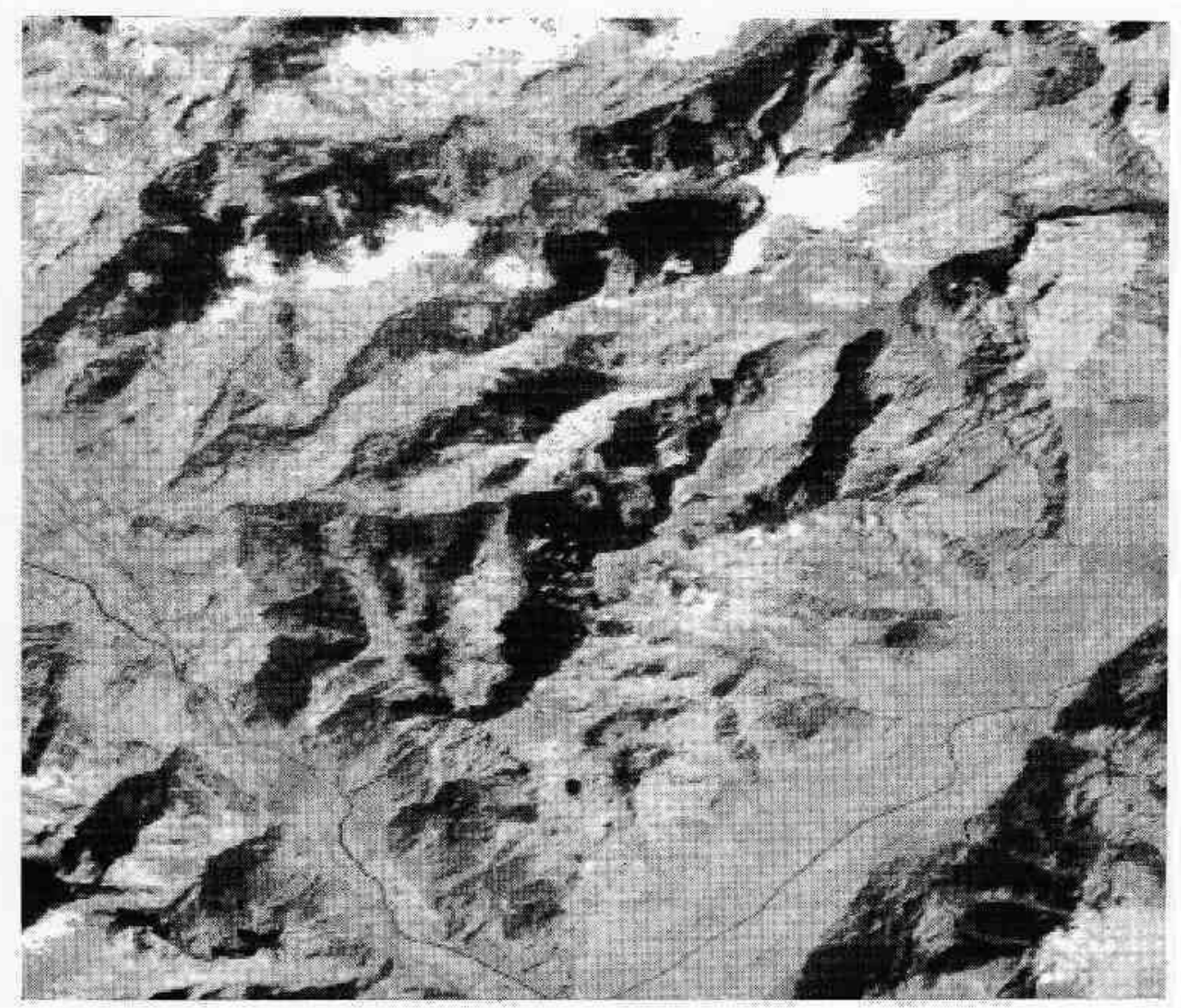

Fia. 6. Original, unrectified image of band $7(0.8$ to $1.1 \mu)$ output of LANDSAT. Notice regular striping.

image data reveals uneven intervals in the analog-to-digital converters used, which contribute to this effect (in fact some codes near major transitions never occur). The relative smoothness of the lookup tables used in destriping this band, shown in Fig. 8, is in part due to the fact that no compression and decompression is performed on data for this band; six-bit numbers linearly related to sensor output are transmitted directly. The inverse transfer functions shown in Fig. 8 appear to be fairly linear, which is probably a result of the linearity of the silicon photodiodes used for this infrared band. One would expect then that the simple destriping method would be fairly successful for this band.

We experienced some difficulties due to the normalization processing performed on the raw LANDSAT data. It may be useful to provide users of LANDSAT tapes optionally with the original data. Preliminary results indicate that slightly better destriping may be possible using the raw image sensor values. It is also unfortunate that areas of high reflectance produce scene radiances which saturate the imaging system. If this was not the case, image sensor outputs corresponding to areas covered by thick clouds could be used in calibration of absolute reflectances as well as in normalization for destriping purposes. 


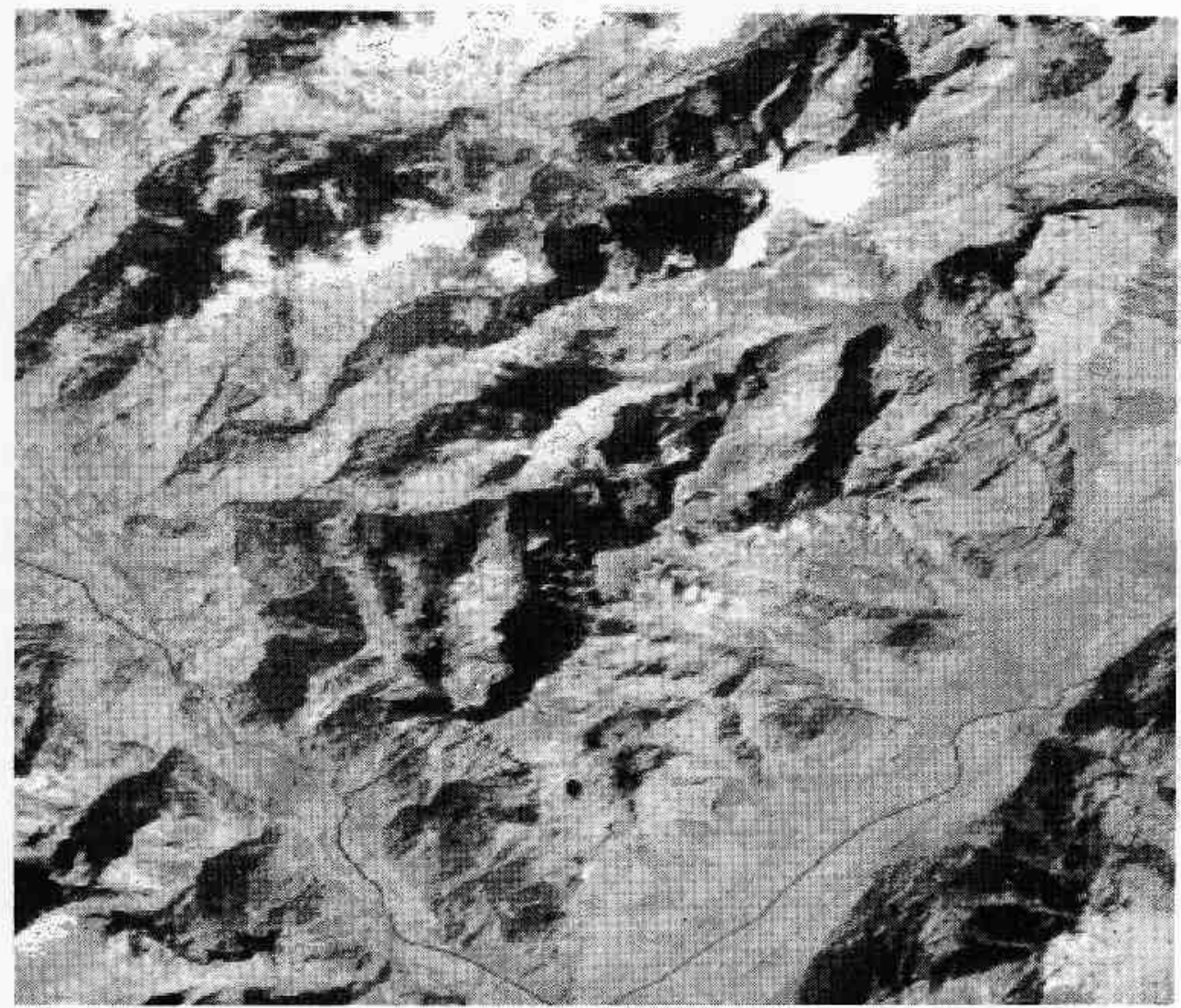

FIG. 7. Destriped image of band 7 output.

\section{RELATION TO HISTOGRAM NORMALIZATION METHODS}

A number of techniques aimed at enhancement of images intended for human viewing are based on manipulation of the gray level histogram. Some, for example, transform the histogram into one considered more desirable [5-11], either flat or "hyperbolic." The suggestion has been made that such techniques allow for changes in sensor characteristics or scene illumination $[6,8,12]$. Several suggestions have been made regarding the difficulty indicated in Fig. 2 relating to the mismatch of two cumulative histograms. One can, for example, inerease the apparent fineness of the quantization by taking into account the context of each picture cell $[7]$. If each picture cell has a maximum gray level value $m$, and has $k$ neighbors, then one may multiply its gray level value by $m k$ and subtract (or add) the sum of the neighboring gray level values. In this fashion rank-ordering of picture cell values is preserved, while the number of possible gray level values is vastly expanded, making the resulting distributions more similar to those found in the eontinuous case.

It is a pity, by the way, that the term "histogram equalization" has come to mean "histogram flattening"; that is, making a histogram uniform $[8,10,11]$. 
a.

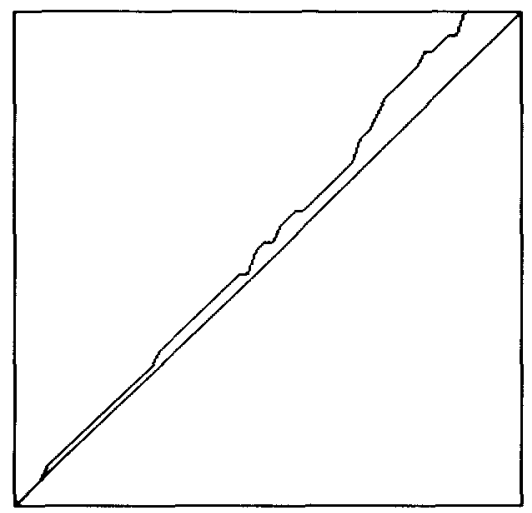

c.
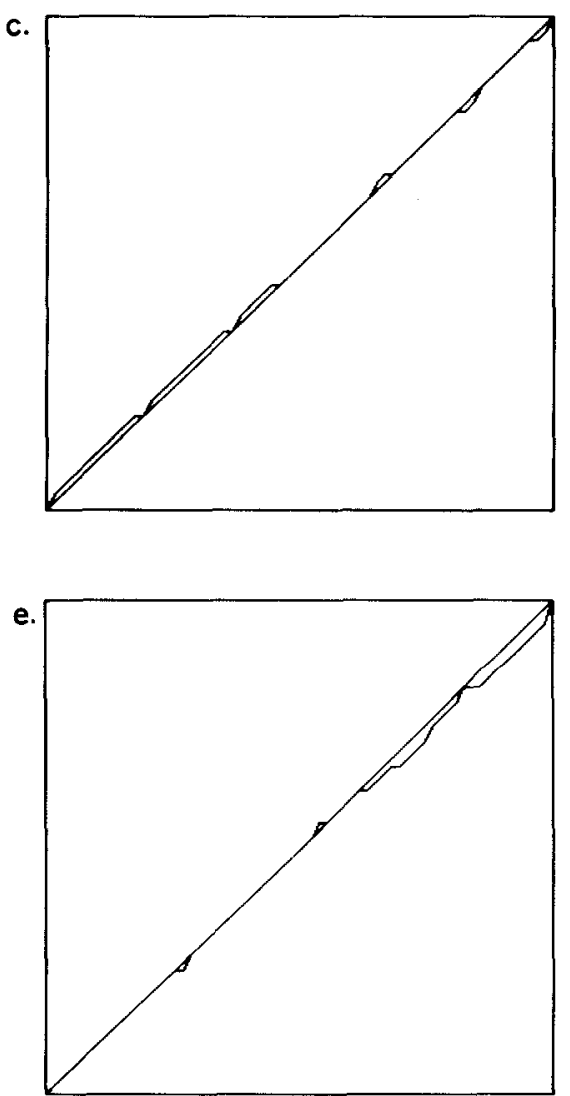

b.

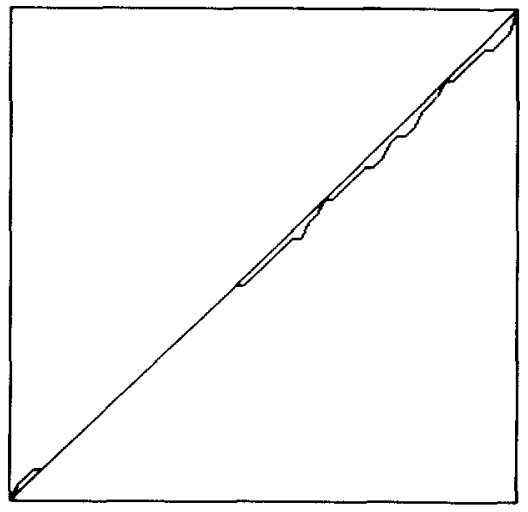

d.

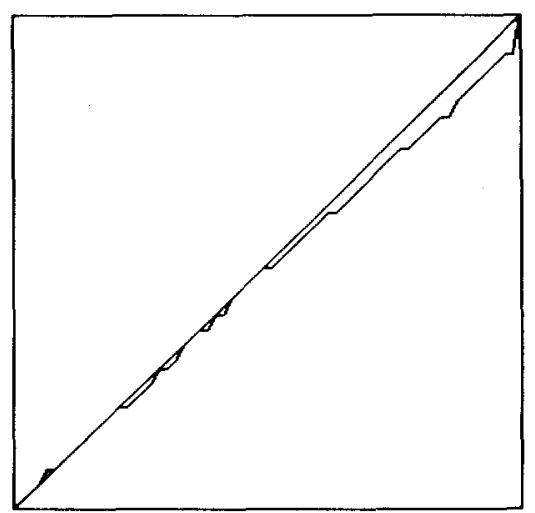

f.

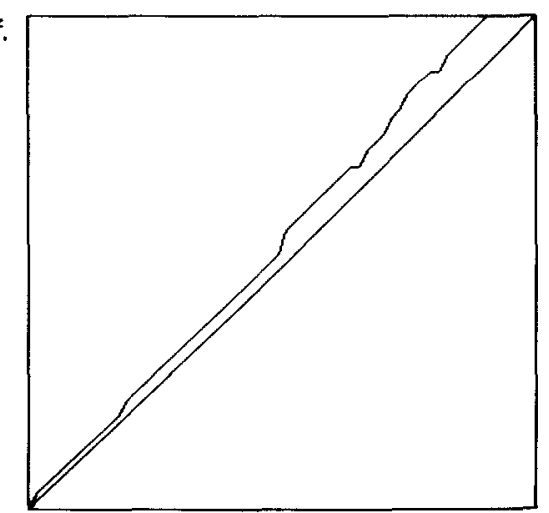

FIG. 8. The destriping lookup tables for band 7-inverse transfer functions for the six image sensors. These transducers appear to be fairly linear, differing mostly only as regards gain or amplification.

It would have been an appropriate term to describe the method presented here where two histograms are made (nearly) equal.

Recent work on matching of images obtained by the same sensor at different 
times is related to our work here on destriping [12]. This sort of idea has, however, not yet found much of a following in the remote sensing community [13]. After this paper was submitted for publication our attention was drawn to two unpublished reports describing similar attempts at destriping of satellite images $[14,15]$. Robinson and Frei suggest equalization [11] or hyperbolization [9] of histograms of subimages $[14$, p. 27]. While this will remove the striping it also will change the tone scale of the image. This may not be desirable when the result is to be used in further machine analysis rather than for presentation to human viewers.

Goetz et al. suggest forcing the sensor histogram to resemble the "average" histogram by a nonlinear transformation [15, p. 17]. Their apparent dissatisfaction with this technique may be traced to the fact that it was applied to complete LANDSAT MSS images. Unfortunately the characteristics of the sensing elements change with time and exposure history, making it impossible to achieve good destriping using a constant lookup table for correction. Better results are achieved with sections of images as shown here. Naturally the sections cannot be made too small, since the estimates of the gain functions would become too unreliable. One cannot say what the optimum subimage size is without knowing more about the statistical properties of the drift of the gain functions of the sensors. It seems reasonable to consider schemes which use slowly varying estimates of the gain functions obtained from windows of the image surrounding the area currently being processed. All these complications can be avoided by using stable, linear sensors.

\section{CONCLUSIONS}

The following is accomplished by the methods presented here:

1. The effect of differences in the transfer functions of the different sensors is removed. All gray levels are then related in the same way to the original scene radiance values.

2. The overall tone scale is preserved. That is, the histogram of gray levels of the destriped image is (approximately) the same as the histogram of the raw image. There is no loss in resolution, nor is the noise level increased.

3 . The computation requires only two serial passes over the whole image: one to collect the relevant histograms, another to apply the inverse transducer function represented as a simple lookup table.

\section{ACKNOWLEDGMENTS}

The authors wish to acknowledge the help of Karen Prendergast in preparing the figures and Meyer Billmers in preparing the computer-generated halftones of the satellite images.

This report describes research done at the Artificial Intelligence Laboratory of the Massachusetts Institute of Technology. Support for the laboratory's artificial intelligence research is provided in part by the Advanced Research Projects Agency of the Department of Defense under Office of Naval Research contract No. N00014-75-C-0643.

\section{REFERENCES}

1. LANDSAT Data Users Handbook, NASA Document No. 76SDS4258, Goddard Space Flight Center, Greenbelt, Md., September 1976. 
2. W. M. Strome and S. S. Vishnubhatla, A system for improving the radiometric corrections for ERTS-1 MSS data, in "XXIV Int. Astronautical Congress, Baku, USSR, October 1973."

3. D. S. Sloan and R. Orth, A self-contained LANDSAT data reception and precision cartographic image production system, in "Int. Symp. of Comm. III of the Int. Soc. for Photogrammetry, Graz, Austria, October 3-5, 1977."

4. D. E. Knuth, The Art of Computer Programming, Vol. 2, pp. 102-103. Addison-Wesley, Reading, Mass., 1969.

5. E. H. Hall, Almost uniform distributions for computer image enhancement, IEEE Trans. Computers C-23, 1974, 207-208.

6. R. M. Haralick, Automatic remote sensor image processing, in "Digital Picture Analysis" (A. Rosenfeld, Ed.), p. 16, Springer, Berlin, 1976.

7. R. A. Hummel, Histogram modification techniques, Computer Graphics and Image Processing 4, 1975, 209-224.

8. A. Rosenfeld and A. C. Kak, "Digital Picture Processing," pp. 173-176. Academic Press, New York, 1976.

9. W. Frei, Image enhancement by histogram hyperbolization, Computer Graphics and Image Processing 6, 1977, 286-294.

10. R. C. Gonzalez and P. A. Wintz, "Digital Image Processing," pp. 118-136. Addison-Wesley, Reading, Mass., 1977.

11. R. Hummel, Image Enhancement by Histogram Transformation, Computer Graphics and Image Processing 6, 1977, 184-195.

12. We-Min Chow and L. T. Kou, Matching two digital pictures, IBM Research Report RC 6870, November 1977.

13. J. Lintz, Jr. and D. S. Simonett (Eds.), "Remote Sensing of the Environment," AddisonWesley, Reading, Mass., 1976.

14. G. S. Robinson and W. Frei, "Final Research Report on Computer Processing of ER'TS Images," USC-IPI Report 640, pp. 22-27. Image Processing Institute, University of Southern California, Los Angeles, September 1975.

15. A. F. H. Goetz et al., "Application of ERTS Images and Image Processing to Regional Geologic Problems and Geologic Mapping in Northern Arizona," pp. 14う̃ 149. TR 32-1597, Jet Propulsion Laboratory, Pasadena, Calif., May 1975. 